NUMER. FUNCT. ANAL. AND OPTIMIZ., 22(7\&8), 861-884 (2001)

\title{
A MANN ITERATIVE REGULARIZATION METHOD FOR ELLIPTIC CAUCHY PROBLEMS
}

\author{
H. W. Engl and A. Leitão*,† \\ Institut für Industriemathematik, Johannes Kepler \\ Universität, A-4040 Linz, Austria
}

\begin{abstract}
We investigate the Cauchy problem for linear elliptic operators with $C^{\infty}$-coefficients at a regular set $\Omega \subset \mathbb{R}^{2}$, which is a classical example of an ill-posed problem. The Cauchy data are given at the manifold $\Gamma \subset \partial \Omega$ and our goal is to reconstruct the trace of the $H^{1}(\Omega)$ solution of an elliptic equation at $\partial \Omega / \Gamma$. The method proposed here composes the segmenting Mann iteration with a fixed point equation associated with the elliptic Cauchy problem. Our algorithm generalizes the iterative method developed by Maz'ya et al., who proposed a method based on solving successive well-posed mixed boundary value problems. We analyze the regularizing and convergence properties both theoretically and numerically.
\end{abstract}

\section{INTRODUCTION}

The solution of a linear elliptic Cauchy problem is written as the solution of a fixed point equation for an affine operator $T$ (see Section 2). This fixed point equation is obtained as a byproduct in [Le].

*Corresponding author. E-mail: aleitao@mtm.ufsc.br. On leave from Department of Mathematics, Federal University of Santa Catarina, P.O. Box 476, 88010-970 Florianópolis, Brazil.

${ }^{\dagger}$ Supported by CNPq under grant 200049-94.1 and FWF project F-1308 within Spezialforschungsbereich 13 . 
In Section 3.1 we discuss the functional analytical formulation in [Le] of an iterative method for solving elliptic Cauchy problems, which was originally proposed by Maz'ya et al. in [KMF].

The original formulation of the Mann iteration (see [Ma]) is discussed in Section 3.2. We also analyze a variant introduced by Groetsch, called segmenting Mann iteration (see [Gr], [EnSc]). These iterative methods approximate the solution of fixed point equations. The Groetsch variation is particularly interesting, when the fixed point equation is described by a non-expansive operator.

In Section 4 we formulate the segmenting Mann iteration for the fixed point equation of Section 2, associated with the solution of an elliptic Cauchy problem. A convergence proof for this new method is given in Section 4.1. Basically we choose a special topology for the space of boundary data, which allow us to prove uniform convexity and to verify some spectral properties of $T$. These properties are the cornerstone of the proof. A regularization property of the method is proved in Section 4.3. Then we provide convergence rates for this regularization method and report about its numerical performance in Section 5.

\section{ELLIPTIC CAUCHY PROBLEMS AND FIXED POINT EQUATIONS}

Let $\Omega \subset \mathbb{R}^{2}$ be an open bounded set and $\Gamma \subset \partial \Omega$ a given manifold. We denote by $P$ a second order elliptic operator defined in $\Omega$. We denote by elliptic Cauchy problem the following (time independent) initial value problem for the operator $P$

$$
\begin{cases}P u=0 & \text { in } \Omega \\ u=f & \text { at } \Gamma \\ u_{v}=g & \text { at } \Gamma\end{cases}
$$

where the given functions $f, g: \Gamma \rightarrow \mathbb{R}$ are called Cauchy data.

The problem we want to solve is to evaluate the trace of the solution of such an initial value problem at the part of the boundary where no data is prescribed, i.e. at $\partial \Omega \backslash \Gamma$. As a solution of the Cauchy problem (CP) we consider a $H^{1}(\Omega)$-distribution, which solves the weak formulation of the elliptic equation in $\Omega$ and also satisfies the Cauchy data at $\Gamma$ in the sense of the trace operator.

It is well known that elliptic Cauchy problems are not well posed in the sense of Hadamard. ${ }^{1}$ A famous example given by Hadamard himself (see [Had]) shows that we cannot have continuous dependence of the data. Also existence of solutions for arbitrary Cauchy data $(f, g)$ cannot be

\footnotetext{
${ }^{1}$ For a formal definition of well posed problems, see e.g. [Ba] or [EHN].
} 
assured, ${ }^{2}$ as shows a simple argumentation with the Schwartz reflection principle (see [GiTr]). However, extending the Cauchy-Kowalewsky and Holmgren Theorems to the $H^{1}$-context, it is possible to prove uniqueness of solutions in weak sense (see [DaLi], [Is]).

Our next step is to characterize the solution of $(\mathrm{CP})$ as solution of a fixed point equation. We define $\Gamma_{1}:=\Gamma, \Gamma_{2}:=\partial \Omega \backslash \Gamma$, such that $\Gamma_{1} \cap \Gamma_{2}=\varnothing$ and $\overline{\Gamma_{1} \cup \Gamma_{2}}=\partial \Omega$. Further, we make the following assumption on the second order elliptic differential operator $P$

$$
P(u):=-\sum_{i, j=1}^{2} D_{i}\left(a_{i, j} D_{j} u\right),
$$

where the operator coefficients $a_{i, j}$ satisfy

- $a_{i, j} \in L_{\infty}(\Omega)$;

- the matrix $A(x):=\left(a_{i, j}\right)_{i, j=1}^{2}$ satisfies: $\xi^{t} A(x) \xi>\alpha\|\xi\|^{2}$, a.e. $x \in \Omega$, $\forall \xi \in \mathbb{R}^{2}$, where $\alpha>0$ is given (independent of $x$ ).

Given the Cauchy data $(f, g) \in H^{1 / 2}\left(\Gamma_{1}\right) \times H_{00}^{1 / 2}\left(\Gamma_{1}\right)^{\prime}$, we assume that there exists a $H^{1}$-solution of the problem ${ }^{3}$

$$
P u=0 \quad \text { in } \Omega, \quad u=f \quad \text { at } \Gamma_{1}, \quad u_{v_{A}}=g \text { at } \Gamma_{1} .
$$

We are mainly interested in the determination of the Neumann trace

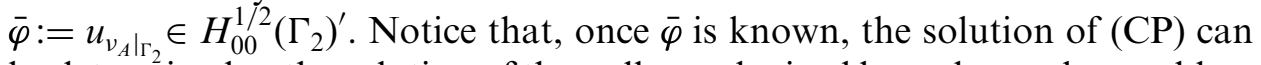
be determined as the solution of the well posed mixed boundary value problem

$$
P u=0 \quad \text { at } \Omega, \quad u=f \quad \text { at } \Gamma_{1}, \quad u_{v_{A}}=\bar{\varphi} \text { at } \Gamma_{2} .
$$

Next we define the operators $L_{n}: H_{00}^{1 / 2}\left(\Gamma_{2}\right)^{\prime} \rightarrow H^{1 / 2}\left(\Gamma_{2}\right), L_{d}: H^{1 / 2}\left(\Gamma_{2}\right) \rightarrow$ $H_{00}^{1 / 2}\left(\Gamma_{2}\right)^{\prime}$ by $L_{n}(\varphi):=w_{\left.\right|_{\Gamma_{2}}}, L_{d}(\psi):=v_{\left.v_{A}\right|_{\Gamma_{2}}}$, where $w, v \in H^{1}(\Omega)$ solve

$$
P w=0 \quad \text { in } \Omega, \quad w=f \quad \text { at } \Gamma_{1}, \quad w_{v_{A}}=\varphi \quad \text { at } \Gamma_{2}
$$

and

$$
P v=0 \quad \text { in } \Omega, \quad v_{v_{A}}=g \text { at } \Gamma_{1}, \quad v=\psi \quad \text { at } \Gamma_{2}
$$

respectively. Now, defining the operator

$$
T: H_{00}^{1 / 2}\left(\Gamma_{2}\right)^{\prime} \ni \varphi \mapsto L_{d}\left(L_{n}(\varphi)\right) \in H_{00}^{1 / 2}\left(\Gamma_{2}\right)^{\prime}
$$

and observing that

$$
L_{n}(\bar{\varphi})=u_{\Gamma_{2}}, \quad L_{d}\left(u_{\Gamma_{2}}\right)=\bar{\varphi}
$$

we obtain the desired characterization $T(\bar{\varphi})=\bar{\varphi}$.

${ }^{2}$ The Cauchy data $(f, g)$ is called consistent if the corresponding problem $(\mathrm{CP})$ has a solution. Otherwise $(f, g)$ is called inconsistent Cauchy data.

${ }^{3}$ For details on the definition of the Sobolev spaces see [Ad] or [DaLi]. 
Note that $T$ is an affine operator, since $L_{n}$ and $L_{d}$ are affine as well. The linear part of $T$ is denoted by $T_{l} \in \mathcal{L}\left(H_{00}^{1 / 2}\left(\Gamma_{2}\right)^{\prime}\right)$. The affine term of the operator $T$ depends on the Cauchy data $(f, g)$ and is denoted by $z_{f, g} \in H_{00}^{1 / 2}\left(\Gamma_{2}\right)^{\prime}$. Than we conclude that the solution $\bar{\varphi}$ of $(\mathrm{CP})$ is also a solution of the fixed point equation

$$
T \varphi\left(=T_{l} \varphi+z_{f, g}\right)=\varphi .
$$

The converse is also true, i.e. if $\bar{\varphi}$ is a solution of (3), one concludes from the uniqueness of solution of $(\mathrm{CP})$ that $\bar{\varphi}$ must be equivalent to $u_{\Gamma_{\Gamma_{2}}}$.

\section{ITERATIVE METHODS}

\subsection{The Maz'ya Iteration}

In this section we discuss the functional analytical formulation in [Le] of an iterative method originally proposed by Maz'ya et al. (see [KMF]). Defining $\mathcal{H}:=H_{00}^{1 / 2}\left(\Gamma_{2}\right)^{\prime}$ and following the notation of Section 2, the Maz'ya iteration can be written in the form of the algorithm:

1. Choose $\varphi_{1} \in \mathcal{H}$;

2. For $k=1,2, \ldots$, do

$$
\begin{aligned}
& \psi_{k}:=L_{n}\left(\varphi_{k}\right) ; \\
& \varphi_{k+1}:=L_{d}\left(\psi_{k}\right) ;
\end{aligned}
$$

Notice that this is equivalent to set $\varphi_{k+1}:=T \varphi_{k}, k=0,1, \ldots$, which is exactly the Picard successive approximation for equation (3). In this particular case we have

$$
\varphi_{k+1}=T^{k}\left(\varphi_{1}\right)=T_{l}^{k}\left(\varphi_{1}\right)+\sum_{j=0}^{k-1} T_{l}^{j}\left(z_{f, g}\right) .
$$

The choice of a special topology for the Hilbert space $\mathcal{H}$ allows one to verify the non-expansivity and asymptotic regularity of the operator $T_{l}{ }^{4}$ These are the key properties used in [Le] to prove the strong convergence of the sequence $\left\{\varphi_{k}\right\}$ to the solution $\bar{\varphi}$ of problem (CP). The convergence of the Maz'ya iteration follows basically from

Lemma 1. Let $H$ be a Hilbert space and $A: H \rightarrow H$ a linear non-expansive regular asymptotic operator. Given $x \in H$, the sequence $\left\{A^{k} x\right\}$ converges to the orthogonal projection of $x$ onto $\operatorname{Ker}(I-A) .^{5}$

The interpretation of the Cauchy problem's solution as a solution of a fixed point equation is already suggested in $[\mathrm{KMF}]$. In this paper, Maz'ya

\footnotetext{
${ }^{4}$ The corresponding definitions can be found in [BrPe].

${ }^{5}$ See [Je] for a complete proof.
} 
et al. based their argumentation on some monotonicity results and elliptic theory to prove the convergence of the iterative method.

\subsection{The Mann Iteration}

In this section we analyze the iterative method introduced by Mann (see [Ma]) to determine solutions of fixed point equations. Let $X$ be a Banach space and $E \subset X$ a convex compact subset. Given a continuous operator $T: E \rightarrow E$, we know from Schauder's fixed point theorem that $T$ has at least one fixed point in $E$. The considered task is that of constructing in $E$ a sequence that converges to a fixed point of $T$.

The starting point for the development of the Mann iteration is the ordinary iteration process $x_{k+1}:=T\left(x_{k}\right)$, with $x_{1} \in E$ arbitrarily chosen. ${ }^{6}$ Let us introduce the infinite triangular matrix

$$
A=\left(\begin{array}{cccccc}
1 & 0 & 0 & \cdots & 0 & \cdots \\
a_{21} & a_{22} & 0 & \cdots & 0 & \cdots \\
a_{31} & a_{32} & a_{33} & \cdots & 0 & \cdots \\
\vdots & \vdots & \vdots & \ddots & \vdots & \vdots
\end{array}\right),
$$

where the coefficients $a_{i j}$ satisfy

$$
\text { i) } a_{i j} \geq 0, \quad i, j=1,2, \ldots \quad \text { ii) } a_{i j}=0, j>i \quad \text { iii) } \sum_{j=1}^{i} a_{i j}=1, \quad i=1,2, \ldots
$$

The Mann iteration is defined by

1. Choose $x_{1} \in E$;

2. For $k=1,2, \ldots$ do

$$
\begin{aligned}
& v_{k}:=\sum_{j=1}^{k} a_{k j} x_{j} ; \\
& x_{k+1}:=T\left(v_{k}\right) ;
\end{aligned}
$$

This method is denoted briefly by $M\left(x_{1}, A, T\right)$. It can be regarded as a generalization of the ordinary iteration process, since this corresponds to the special choice $A=I$ (the identity matrix). In the early work [Ma], Mann proves the following result:

Lemma 2. If either of the sequences $\left\{x_{k}\right\},\left\{v_{k}\right\}$ converges, than the other also converges to the same limit, and this common limit is a fixed point of $T$.

Further properties of the sets of limit points of $\left\{x_{k}\right\}$ and $\left\{v_{k}\right\}$ are also proved, under additional requirements on the coefficients $a_{i j}$.

\footnotetext{
${ }^{6}$ It is obvious that this Picard iteration may fail to converge in this general framework.
} 
The proof in [Ma] can be extended to a locally convex Hausdorff vector space $X$ and $E \subset X$ a convex closed subset, by using the regularity of the matrix $A$. A sketch of the proof is given in [Do], which also analyzes the Mann iteration for quasi non-expansive operators.

In the article [Gr], Groetsch considers a variant of the iteration defined above. The Banach space $X$ is assumed to be uniformly convex and $E \subset X$ convex only. Additionally to $i$ ), ii) and iii), the further assumption

$$
\text { iv) } a_{i+1, j}=\left(1-a_{i+1, i+1}\right) a_{i j}, j \leq i
$$

is made, in which case the matrix $A$ is said to be segmenting. ${ }^{7}$ The corresponding iterative method is called Mann segmenting iteration. As one can easily check, $v_{k+1}$ can be written in this case as the convex linear combination

$$
v_{k+1}=\left(1-d_{k}\right) v_{k}+d_{k} T\left(v_{k}\right),
$$

where $d_{k}:=a_{k+1, k+1}$, i.e. $v_{k+1}$ lies on the line segment joining $v_{k}$ and $x_{k+1}=$ $T\left(v_{k}\right)$. Notice that the choice of the diagonal elements $d_{k}$ determines completely the segmenting matrix $A$. Next we enunciate the main theorem in [Gr]:

Lemma 3. Let $T$ be a non-expansive operator with at least one fixed point in $E$. If $\sum_{k=1}^{\infty} d_{k}\left(1-d_{k}\right)$ diverges, then the sequence $\left\{(I-T) v_{k}\right\}$ converges strongly to zero, for every $x_{1} \in E$.

Notice that, differently from Lemma 2, compactness of $E$ is not required in Lemma 3. This is the reason, why the existence of fixed points of $T$ must be assumed in the last lemma.

In order to prove that $M\left(x_{1}, A, T\right)$ converges strongly to a fixed point of $T$ for every $x_{1} \in E$, one needs stronger assumptions such as:

- $E$ is closed convex; $T(E)$ is relatively compact in $X$.

- $E$ is closed convex; $I-T$ maps bounded closed subsets of $E$ into closed subsets of $E$.

- $E$ is closed convex; $T$ is demicompact in the sense of $[\mathrm{BrPe}]$.

One should note that the last condition is a particular case of the second one.

In the particular case $X$ is an Euclidian space, $E \subset X$ is a convex compact subset, $T: E \rightarrow E$ is a non-expansive mapping with a unique fixed point $x \in E$, and $A$ is a segmenting matrix such that $\sum_{k=1}^{\infty} d_{k}\left(1-d_{k}\right)$ diverges, then $M\left(x_{1}, A, T\right)$ converges to $x$, for every $x_{1} \in E$.

It is worth mentioning that Lemma 3 gives on $M\left(x_{1}, A, T\right)$ a condition analogous to the asymptotic regularity, which was used in Lemma 1 to prove the convergence of the Maz'ya iteration.

${ }^{7}$ Due to the geometrical interpretation, we adopt the notation used in [Gr] and [EnSc]. In [Do], matrices satisfying $i v$ ) are called normal matrices. 


\section{AN ITERATIVE METHOD FOR CAUCHY PROBLEMS}

In this section we introduce a segmenting Mann iteration for solving the elliptic Cauchy problem (CP). Let $T$ be the operator defined in (2) and $A$ a segmenting matrix. We assume the Cauchy data $(f, g)$ are consistent and denote by $\bar{\varphi}$ the solution of the fixed point equation (3). The MannMaz'ya iteration is defined by:

1. Choose $\varphi_{1} \in \mathcal{H}$;

2. For $k=1,2, \ldots$ do

$$
\begin{aligned}
& \phi_{k}:=\sum_{j=1}^{k} a_{k j} \varphi_{j} ; \\
& \varphi_{k+1}:=T\left(\phi_{k}\right) ;
\end{aligned}
$$

Note that $\varphi_{k}, \phi_{k} \in \mathcal{H}, k=1,2, \ldots$. We represent this iterative process by $\left(\varphi_{1}, A, T\right)$. Obviously it coincides with the Maz'ya iteration if one chooses $A=I$.

Defining the iteration errors $\varepsilon_{k}:=\varphi_{k}-\bar{\varphi}$ and $\gamma_{k}:=\phi_{k}-\bar{\varphi}$, we obtain

$$
\begin{aligned}
& \varepsilon_{k+1}=\varphi_{k+1}-\bar{\varphi}=T\left(\phi_{k}\right)-T(\bar{\varphi})=T_{l}\left(\phi_{k}-\bar{\varphi}\right)=T_{l}\left(\gamma_{k}\right), \\
& \gamma_{k}=\phi_{k}-\bar{\varphi}=\sum_{j=1}^{k} a_{k j} \varphi_{j}-\bar{\varphi}=\sum_{j=1}^{k} a_{k j} \varphi_{j}-\sum_{j=1}^{k} a_{k j} \bar{\varphi}=\sum_{j=1}^{k} a_{k j} \varepsilon_{j} .
\end{aligned}
$$

It becomes clear, that the convergence of the iteration $\left(\varphi_{1}, A, T\right)$ to the fixed point $\bar{\varphi}$ is equivalent to the convergence (to zero) of the iteration $\left(\varepsilon_{1}, A, T_{l}\right)$.

In order to analyze this method, we need to define a special topology for the space $\mathcal{H}$.

Definition 4. Given $\varphi \in \mathcal{H}$, let $W(\varphi) \in H^{1}(\Omega)$ be the solution of the mixed boundary value problem

$$
P w=0 \quad \text { in } \Omega, \quad w=0 \quad \text { at } \Gamma_{1}, \quad w_{v_{A}}=\varphi \text { at } \Gamma_{2} .
$$

We define the functional $\|\cdot\|_{*}: \mathcal{H} \rightarrow \mathbb{R}$ by $\|\varphi\|_{*}:=\left(\int_{\Omega}|\nabla W(\varphi)|^{2} d x\right)^{1 / 2}$.

It is proved in [Le] that the functional $\|\cdot\|_{*}$ defines a norm in $\mathcal{H}$, which is equivalent to the usual Sobolev norm of this space. Actually, one can verify that the bilinear form

$$
\langle\varphi, \psi\rangle_{*}:=\int_{\Omega} \nabla W(\varphi) \nabla W(\psi) d x
$$

defines an inner product in $\mathcal{H}$. In the Hilbert space $\left(\mathcal{H} ;\langle\cdot, \cdot\rangle_{*}\right)$ we are able to analyze the Mann-Maz'ya iteration $\left(\varphi_{1}, A, T\right)$. 


\subsection{Convergence Proof}

We start this section discussing an auxiliary convexity result, that is needed for the proof of the main theorem. ${ }^{8}$

Lemma 5. Let $\varphi_{k}, \phi_{k}$ be the sequences generated by the iteration $\left(\varphi_{1}, A, T\right)$ and $\bar{\varphi}$ the solution of the fixed point equation (3). If for some $\varepsilon>0, k_{0} \in \mathbb{N}$ the inequality $\left\|T\left(\phi_{k}\right)-\varphi_{k}\right\| \geq \varepsilon, \forall k \geq k_{0}$ holds, then there exists $c>0$ such that

$$
\left\|\phi_{k+1}-\bar{\varphi}\right\| \leq\left(1-c d_{k}\left(1-d_{k}\right)\right)\left\|\phi_{k}-\bar{\varphi}\right\|, \quad k \geq k_{0} .
$$

Proof. Notice that $\left\|\phi_{k}-\bar{\varphi}\right\| \leq\left\|\phi_{1}-\bar{\varphi}\right\|$, for $k \geq 1$ (see (11) below). Since the Hilbert space $\mathcal{H}$ is uniformly convex, we obtain for every pair $x, y \in \mathcal{H}$ with $\|x\|,\|y\| \leq\left\|\phi_{k}-\bar{\varphi}\right\|$ and $\|x-y\| \geq \varepsilon$ the inequality

$$
\begin{aligned}
\|\lambda x+(1-\lambda) y\| & \leq 2 \lambda\|(1 / 2)(x+y)\|+(1-2 \lambda)\|y\| \\
& \leq 2 \lambda(1-\tilde{c})\left\|\phi_{k}-\bar{\varphi}\right\|+(1-2 \lambda)\left\|\phi_{k}-\bar{\varphi}\right\| \\
& \leq\left\|\phi_{k}-\bar{\varphi}\right\|[1-2 \lambda \tilde{c}(1-\lambda)], \quad \lambda \in[0,1],
\end{aligned}
$$

where the constant $0<\tilde{c}<1$ depends only on $\varepsilon$ and $\left\|\phi_{1}-\bar{\varphi}\right\|$. Further, it follows from (5)

$$
\phi_{k+1}-\bar{\varphi}=\left(1-d_{k}\right)\left(\phi_{k}-\bar{\varphi}\right)+d_{k}\left(T\left(\phi_{k}\right)-T(\bar{\varphi})\right) .
$$

Now, from (8) and (7) with $x=T\left(\phi_{k}\right)-T(\bar{\varphi}), y=\phi_{k}-\bar{\varphi}, \lambda=d_{k}$, we obtain the inequality in (6) with $c=2 \tilde{c}$.

Next we prove for the Mann-Maz'ya iteration, a result analogous to the one stated Lemma 3.

Theorem 6. Let $T$ be the operator defined in (2) and $A$ a segmenting matrix such that $\sum_{k=1}^{\infty} d_{k}\left(1-d_{k}\right)$ diverges. The iteration $\left(\varphi_{1}, A, T\right)$ generates a sequence $\left\{\phi_{k}\right\}$ such that $\left\{(I-T) \phi_{k}\right\}$ converges strongly to zero, for every $\varphi_{1} \in \mathcal{H}$.

Proof. From the segmenting property (5), follows

$$
\left\|\phi_{k+1}-\phi_{k}\right\|=d_{k}\left\|T\left(\phi_{k}\right)-\phi_{k}\right\| \text {. }
$$

\footnotetext{
${ }^{8}$ For simplicity we denote by $\|\cdot\|$ the norm of the Hilbert space $\mathcal{H}$, but meant is the norm $\|\cdot\|_{*}$
} introduced in Definition 4. 
From the non-expansivity of $T_{l}$ and (9) we obtain

$$
\begin{aligned}
\left\|T\left(\phi_{k+1}\right)-\phi_{k+1}\right\| & \leq\left\|T\left(\phi_{k+1}\right)-T\left(\phi_{k}\right)\right\|+\left\|T\left(\phi_{k}\right)-\phi_{k+1}\right\| \\
& \leq d_{k}\left\|T\left(\phi_{k}\right)-\phi_{k}\right\|+\left\|T\left(\phi_{k}\right)-\left(1-d_{k}\right) \phi_{k}-d_{k} T\left(\phi_{k}\right)\right\| \\
& =d_{k}\left\|T\left(\phi_{k}\right)-\phi_{k}\right\|+\left(1-d_{k}\right)\left\|T\left(\phi_{k}\right)-\phi_{k}\right\| \\
& =\left\|T\left(\phi_{k}\right)-\phi_{k}\right\| .
\end{aligned}
$$

Denoting by $\bar{\varphi}$ the solution of the fixed point equation (3), we estimate

$$
\begin{aligned}
\left\|\phi_{k+1}-\bar{\varphi}\right\| & =\left\|\left(1-d_{k}\right) \phi_{k}+d_{k} T\left(\phi_{k}\right)-\left(1-d_{k}\right) \bar{\varphi}-d_{k} \bar{\varphi}\right\| \\
& =\left\|\left(1-d_{k}\right)\left(\phi_{k}-\bar{\varphi}\right)+d_{k}\left(T\left(\phi_{k}\right)-T(\bar{\varphi})\right)\right\| \\
& \leq\left\|\phi_{k}-\bar{\varphi}\right\|
\end{aligned}
$$

and

$$
\left\|\phi_{k}-T\left(\phi_{k}\right)\right\|=\left\|\phi_{k}-\bar{\varphi}+T(\bar{\varphi})-T\left(\phi_{k}\right)\right\| \leq 2\left\|\phi_{k}-\bar{\varphi}\right\| .
$$

Now, let us assume that $(I-T) \phi_{k} \not \nrightarrow 0$ for some $\varphi_{1}$. Since the sequence $\left\|(I-T) \phi_{k}\right\|$ is monotone decreasing by (10), there exists $\varepsilon>0$ and $k_{0} \in \mathbb{N}$ such that

$$
\left\|T\left(\phi_{k}\right)-\phi_{k}\right\| \geq \varepsilon, \quad k \geq k_{0} .
$$

From (12) and (13) follows

$$
\left\|\phi_{k}-\bar{\varphi}\right\| \geq \varepsilon / 2, \quad k \geq k_{0} .
$$

Now we obtain from (13) and Lemma 5

$$
\begin{aligned}
\left\|\phi_{k+1}-\bar{\varphi}\right\| \leq & \left\|\phi_{k}-\bar{\varphi}\right\|-c d_{k}\left(1-d_{k}\right)\left\|\phi_{k}-\bar{\varphi}\right\| \\
\leq & \left\|\phi_{k-1}-\bar{\varphi}\right\|-c d_{k-1}\left(1-d_{k-1}\right)\left\|\phi_{k-1}-\bar{\varphi}\right\| \\
& -c d_{k}\left(1-d_{k}\right)\left\|\phi_{k}-\bar{\varphi}\right\| \\
\leq & \left\|\phi_{k-1}-\bar{\varphi}\right\|-\left\|\phi_{k}-\bar{\varphi}\right\| c\left(d_{k-1}\left(1-d_{k-1}\right)+d_{k}\left(1-d_{k}\right)\right),
\end{aligned}
$$

for $k \geq k_{0}$. Repeating the argumentation we have

$$
\left\|\phi_{k+1}-\bar{\varphi}\right\| \leq\left\|\phi_{k_{0}}-\bar{\varphi}\right\|-c\left\|\phi_{k}-\bar{\varphi}\right\| \sum_{j=k_{0}}^{k} d_{j}\left(1-d_{j}\right) .
$$

This inequality together with (14) imply

$$
\frac{\varepsilon}{2} \leq\left\|\phi_{k_{0}}-\bar{\varphi}\right\|-c \frac{\varepsilon}{2} \sum_{j=k_{0}}^{k} d_{j}\left(1-d_{j}\right)
$$

and we finally obtain

$$
\varepsilon\left[1+c \sum_{j=k_{0}}^{k} d_{j}\left(1-d_{j}\right)\right] \leq 2\left\|\phi_{k_{0}}-\bar{\varphi}\right\|, \quad k \geq k_{0} .
$$

This gives a contradiction, since $\sum_{j=1}^{\infty} d_{j}\left(1-d_{j}\right)$ diverges by hypothesis. 
Notice that to prove Theorem 6 , it is enough to verify that $(I-T) \times$ $\gamma_{k} \rightarrow 0$, for every $\varepsilon_{1} \in \mathcal{H}$. This can be done with minor adaptations in the above proof.

Corollary 7. Let $T$ be the operator defined in (2) and A a segmenting matrix such that $\sum_{k=1}^{\infty} d_{k}\left(1-d_{k}\right)$ diverges. For every $\varphi_{1} \in \mathcal{H}$ the sequences $\left\{\varphi_{k}\right\},\left\{\phi_{k}\right\}$ generated by the iteration $\left(\varphi_{1}, A, T\right)$ satisfy

$$
\lim _{k \rightarrow \infty} \phi_{k}=\bar{\varphi}=\lim _{k \rightarrow \infty} \varphi_{k},
$$

where $\bar{\varphi}$ is the unique determined solution of the fixed point equation (3).

Proof. Since $(f, g)$ are consistent Cauchy data, existence and uniqueness of $\bar{\varphi}$ can be assured (see Section 2). Since $(I-T) \phi_{k}=\left(I-T_{l}\right)\left(\phi_{k}-\bar{\varphi}\right)$, it follows from Theorem 6 that $\lim _{k}\left(I-T_{l}\right)\left(\phi_{k}-\bar{\varphi}\right)=0$ or alternatively $\lim _{k}$ $\operatorname{dist}\left(\phi_{k}-\bar{\varphi}, N\right)=0$, where $N:=\operatorname{Ker}\left(I-T_{l}\right)$. However we know from [Le, Theorem 2.3] that $\operatorname{Ker}\left(I-T_{l}\right)=\{0\}$, from what follows $\phi_{k} \rightarrow \bar{\varphi}$. The second statement follows from $\lim _{k} \varphi_{k}=\lim _{k} T\left(\phi_{k-1}\right)=T(\bar{\varphi})=\bar{\varphi}$.

\subsection{A Remark on Noisy Cauchy Data}

Before analyzing regularization properties and obtaining convergence rates for the Mann-Maz'ya iteration (see Sections 4.3 and 4.4 below), it is necessary to make some considerations about the treatment of noisy Cauchy data.

Let $(f, g)$ be consistent Cauchy data and $z_{f, g} \in \mathcal{H}$ the corresponding affine term of the operator $T$, defined in Section 2. Notice that for every pair of Cauchy data $(\tilde{f}, \tilde{g}) \in H^{1 / 2}\left(\Gamma_{1}\right) \times H_{00}^{1 / 2}\left(\Gamma_{1}\right)^{\prime}$, consistent or not, we can analogously obtain a corresponding affine term $\tilde{z}$. In this section we investigate the following question: Given the measured data $\left(f_{\varepsilon}, g_{\varepsilon}\right)$ in $L^{2}\left(\Gamma_{1}\right) \times$ $H_{00}^{1 / 2}\left(\Gamma_{1}\right)^{\prime}$, with

$$
\left\|f_{\varepsilon}-f\right\|_{L^{2}}+\left\|g_{\varepsilon}-g\right\|_{\left(H_{00}^{1 / 2}\right)^{\prime}} \leq \varepsilon
$$

how can we obtain a corresponding affine term $z_{\varepsilon}$, such that $\left\|z_{f, g}-z_{\varepsilon}\right\| \leq \varepsilon$.

We claim that $z_{\varepsilon}$ can be obtained under the following a priori assumption on the exact Cauchy data: $f \in H^{r}\left(\Gamma_{1}\right), r \geq 1 / 2$. In order to verify this assertion, we first use a smoothing operator $S: L^{2}\left(\Gamma_{1}\right) \rightarrow H^{1 / 2}\left(\Gamma_{1}\right)$ to generate a $\tilde{f}_{\varepsilon}:=S f_{\varepsilon} \in H^{1 / 2}\left(\Gamma_{1}\right)$, satisfying $\left\|f-\tilde{f}_{\varepsilon}\right\|_{1 / 2} \leq \varepsilon^{\prime}$. The existence of such an operator follows from

Lemma 8. Let $f \in H^{r}, r>s>0$. There exists a smoothing operator $S: L^{2} \rightarrow$ $H^{s}$ and a positive function $\gamma$ with $\lim _{x \downarrow 0} \gamma(x)=0$, such that for $\varepsilon>0$ and $f_{\varepsilon} \in L^{2}$ with $\left\|f-f_{\varepsilon}\right\|_{L^{2}} \leq \varepsilon$, we have

$$
\left\|f-S f_{\varepsilon}\right\|_{s} \leq \gamma(\varepsilon) \text {. }
$$


Proof. See [BaLe, Lemma 14].

After smoothing the data $f_{\varepsilon} \in L^{2}\left(\Gamma_{1}\right)$, we finally obtain from the Cauchy data $\left(\tilde{f}_{\varepsilon}, g_{\varepsilon}\right)$ a corresponding $z_{\varepsilon} \in H_{00}^{1 / 2}\left(\Gamma_{1}\right)^{\prime}$ such that $\left\|z_{f, g}-z_{\varepsilon}\right\|<\varepsilon^{\prime}$ (note that the affine term $z_{f, g}$ depends continuously on the data $\left.(f, g)\right)$.

\subsection{A Regularization Property}

In this section we analyze a regularization property of the MannMaz'ya iteration. We start defining, for every fixed $\varphi \in \mathcal{H}$, the family of operators

$$
R_{k}^{\varphi}: \mathcal{H} \ni \psi \mapsto \varphi_{k} \in \mathcal{H}, \quad k \in \mathbb{N},
$$

where $\varphi_{k}$ is $k$-th element of the sequence generated by the Mann-Maz'ya algorithm $\left(\varphi, A, T_{l}+\psi\right)$. In the next theorem we prove a regularization property of the family $\left\{R_{k}^{\varphi}\right\}_{k}$ with respect to the solution $\bar{\varphi}$ of the fixed point equation (3).

Theorem 9. Let $\varphi$ be an arbitrary element of $\mathcal{H}$ and $\left\{R_{k}^{\varphi}\right\}_{k \in \mathbb{N}}$ be the family of operators defined in (15). There exists $\varepsilon_{0}>0$ and functions $\tau:\left(0, \varepsilon_{0}\right) \rightarrow \mathbb{R}^{+}$, $k:\left(0, \varepsilon_{0}\right) \rightarrow \mathbb{N}$, such that

i) $\tau(\varepsilon) \rightarrow 0$, for $\varepsilon \rightarrow 0$;

ii) For every pair of Cauchy data $\left(f_{\varepsilon}, g_{\varepsilon}\right)$ with $\left\|z_{\varepsilon}-z_{f, g}\right\| \leq \varepsilon$, we have

$$
\left\|R_{k(\varepsilon)}^{\varphi}\left(z_{\varepsilon}\right)-\bar{\varphi}\right\| \leq \tau(\varepsilon) .
$$

Proof. Let the Cauchy data $\left(f_{\varepsilon}, g_{\varepsilon}\right)$ be given as in ii). From the identity $\bar{\varphi}=R_{k}^{\bar{\varphi}}\left(z_{f, g}\right), k \in \mathbb{N}$ follows

$$
\begin{aligned}
\left\|R_{k}^{\varphi}\left(z_{\varepsilon}\right)-\bar{\varphi}\right\| & =\left\|R_{k}^{\varphi-\bar{\varphi}}\left(z_{\varepsilon}-z_{f, g}\right)\right\| \\
& \leq\left\|R_{k}^{\varphi-\bar{\varphi}}(0)\right\|+\left\|R_{k}^{0}\left(z_{\varepsilon}-z_{f, g}\right)\right\| .
\end{aligned}
$$

Using the facts: $\left\|T_{l}\right\| \leq 1, \sum_{j=1}^{k} a_{k j}=1$, one obtains by induction

$$
\left\|R_{k+1}^{0}(\psi)\right\| \leq k\|\psi\|, \quad k \in \mathbb{N} .
$$

Substituting in (16) we have

$$
\left\|R_{k}^{\varphi}\left(z_{\varepsilon}\right)-\bar{\varphi}\right\| \leq \varepsilon k+\left\|R_{k}^{\varphi-\bar{\varphi}}(0)\right\| .
$$

Now we define for $\varepsilon>0$, the function

$$
\tau(\varepsilon):=2 \inf _{k \in \mathbb{N}}\left\{\varepsilon k+\left\|R_{k}^{\varphi-\bar{\varphi}}(0)\right\|\right\} .
$$

Note that the sequence $\left\{R_{k}^{\varphi-\bar{\varphi}}(0)\right\}$ corresponds to the Mann-Maz'ya algorithm for the (consistent) Cauchy data $(f, g)=(0,0)$ with initial point $\varphi_{1}=\varphi-\bar{\varphi}$. From Corollary 7 follows $\lim _{k \rightarrow \infty} R_{k}^{\varphi-\bar{\varphi}}(0)=0$, and we can conclude $\lim _{\varepsilon \rightarrow 0} \tau(\varepsilon)=0$. 
Given $\varepsilon>0$ we choose $k(\varepsilon) \in \mathbb{N}$ with

$\varepsilon k(\varepsilon)+\left\|R_{k(\varepsilon)}^{\varphi-\bar{\varphi}}(0)\right\|<\tau(\varepsilon)$

(this is possible from the definition of $\tau$ ). The theorem follows now from (17).

As an immediate consequence of Theorem 9, we obtain the following regularization property of the Maz'ya iteration:

Corollary 10. The Maz'ya iteration regularizes the fixed point equation (3), i.e. given $\varphi_{1} \in H_{00}^{1 / 2}\left(\Gamma_{2}\right)^{\prime}$, there exists $\varepsilon_{0}>0$ and functions $\tau:\left(0, \varepsilon_{0}\right) \rightarrow \mathbb{R}^{+}$, $k:\left(0, \varepsilon_{0}\right) \rightarrow \mathbb{N}$, such that $\lim _{\varepsilon \rightarrow 0} \tau(\varepsilon)=0$ and for every pair of Cauchy data $\left(f_{\varepsilon}, g_{\varepsilon}\right)$ with $\left\|z_{\varepsilon}-z_{f, g}\right\| \leq \varepsilon$ the inequality $\left\|\varphi_{k(\varepsilon)}-\bar{\varphi}\right\| \leq \tau(\varepsilon)$ holds.

\subsection{Convergence Rates}

Notice that in Theorem 9, we obtain an estimate for the iteration error $\varepsilon_{k}=\varphi_{k}-\bar{\varphi}$. However, without making any further assumption on $\varepsilon_{0}$ (or equivalently on $\bar{\varphi}$ ), we cannot give concrete choices for $\tau(\cdot), k(\cdot)$ as functions of $\varepsilon$, i.e. we cannot prove convergence rates.

In this section we consider the iteration residual and use the discrepancy principle as stopping rule (again without any additional regularity assumption on $\bar{\varphi}$ ), in order to obtain a similar (but constructive) result (at least for the residuals; for rates of convergence for the iterates themselves, we will need additional conditions as explained in Section 4.5).

We start by defining the iteration residual. Let $(f, g)$ be consistent Cauchy data and $z_{\varepsilon} \in \mathcal{H}$ with $\left\|z_{\varepsilon}-z_{f, g}\right\| \leq \varepsilon$. Given $\varphi_{1} \in \mathcal{H}$, let us consider the sequences

$$
\varphi_{k+1}=T_{l} \varphi_{k}+z_{f, g}, \quad \varphi_{k+1}^{\varepsilon}=T_{l} \varphi_{k}^{\varepsilon}+z_{\varepsilon}, \quad k=1,2, \ldots,
$$

$\varphi_{1}^{\varepsilon}=\varphi_{1}$. The corresponding residuals (exact and real, i.e. using noisy data) are defined by

$$
r_{k}:=z_{f, g}-\left(I-T_{l}\right) \varphi_{k}, \quad r_{k}^{\varepsilon}:=z_{\varepsilon}-\left(I-T_{l}\right) \varphi_{k}^{\varepsilon} .
$$

Now let $\mu>1$ be fixed. According to the discrepancy principle, we should stop the iteration at the step $k\left(\varepsilon, z_{\varepsilon}\right)$ when for the first time $\left\|r_{k\left(\varepsilon, z_{\varepsilon}\right)}^{\varepsilon}\right\| \leq \mu \varepsilon$, i.e.

$$
k\left(\varepsilon, z_{\varepsilon}\right):=\min \left\{k \in \mathbb{N} \mid\left\|z_{\varepsilon}-\left(I-T_{l}\right) \varphi_{k}^{\varepsilon}\right\| \leq \mu \varepsilon\right\} .
$$

Remark 11. Notice that the residual sequences $\left\{\left\|r_{k}^{\varepsilon}\right\|\right\},\left\{\left\|r_{k}\right\|\right\}$ are nonincreasing. Indeed, this follows from

$$
\begin{aligned}
z_{f, g}-\left(I-T_{l}\right) \varphi_{k+1} & =z_{f, g}-\left(I-T_{l}\right)\left(T_{l} \varphi_{k}+z_{f, g}\right) \\
& =T_{l}\left(z_{f, g}-\left(I-T_{l}\right) \varphi_{k}\right), \\
z_{\varepsilon}-\left(I-T_{l}\right) \varphi_{k+1}^{\varepsilon} & =T_{l}\left(z_{\varepsilon}-\left(I-T_{l}\right) \varphi_{k}^{\varepsilon}\right) .
\end{aligned}
$$

and the non-expansivity of $T_{l}$. 
Next we obtain an estimate for $k\left(\varepsilon, z_{\varepsilon}\right)$ in (18). For simplicity we consider only the Maz'ya iteration $(A=I)$, being the general case completely analog. (This result is comparable to the one known for the Landweber iteration; see e.g. [EHN, Section 6.1] or [EnSc].)

Theorem 12. If $\mu>1$ is fixed, the stopping rule $k\left(\varepsilon, z_{\varepsilon}\right)$ determined by the discrepancy principle in (18) satisfies $k\left(\varepsilon, z_{\varepsilon}\right)=O\left(\varepsilon^{-2}\right)$.

Proof. Given a linear non-negative operator $T: \mathcal{H} \rightarrow \mathcal{H}$ we have

$$
\langle T \psi, T \psi\rangle=\langle\psi, \psi\rangle-\langle(I-T) \psi,(I-T) \psi\rangle-2\langle(I-T) \psi, T \psi\rangle, \quad \psi \in \mathcal{H} .
$$

Using this identity for $T=T_{l}, \psi=\bar{\varphi}-\varphi_{j}$, we obtain

$$
\begin{aligned}
\left\|\bar{\varphi}-\varphi_{j+1}\right\|^{2}= & \left\|\bar{\varphi}-\varphi_{j}\right\|^{2}-\left\|z_{f, g}-\left(I-T_{l}\right) \varphi_{j}\right\|^{2} \\
& -2\left\langle\left(I-T_{l}\right)\left(\bar{\varphi}-\varphi_{j}\right), T_{l}\left(\bar{\varphi}-\varphi_{j}\right)\right\rangle
\end{aligned}
$$

(notice that $T \psi=\bar{\varphi}-\varphi_{j+1}$ ) and we can estimate

$$
\begin{aligned}
\left\|\bar{\varphi}-\varphi_{j}\right\|^{2}-\left\|\bar{\varphi}-\varphi_{j+1}\right\|^{2}= & \left\|z_{f, g}-\left(I-T_{l}\right) \varphi_{j}\right\|^{2} \\
& +2\left\langle T_{l}\left(I-T_{l}\right)\left(\bar{\varphi}-\varphi_{j}\right),\left(\bar{\varphi}-\varphi_{j}\right)\right\rangle \\
\geq & \left\|z_{f, g}-\left(I-T_{l}\right) \varphi_{j}\right\|^{2} .
\end{aligned}
$$

Adding up this inequalities for $j=1, \ldots, k$, we obtain

$$
\left\|\bar{\varphi}-\varphi_{1}\right\|^{2}-\left\|\bar{\varphi}-\varphi_{k+1}\right\|^{2} \geq \sum_{j=1}^{k}\left\|z_{f, g}-\left(I-T_{l}\right) \varphi_{j}\right\|^{2} \geq k\left\|z_{f, g}-\left(I-T_{l}\right) \varphi_{k}\right\|^{2}
$$

(in the last inequality we used the monotonicity of the sequence $\left\|r_{k}\right\|$; see Remark 11) and we can conclude

$$
\left\|z_{f, g}-\left(I-T_{l}\right) \varphi_{k}\right\|^{2} \leq k^{-1}\left\|\bar{\varphi}-\varphi_{1}\right\|^{2} .
$$

Now, let us consider the real residual $r_{k}^{\varepsilon}$. We have

$$
\begin{aligned}
\left\|z_{\varepsilon}-\left(I-T_{l}\right) \varphi_{k+1}^{\varepsilon}\right\| & =\left\|T_{l}^{k}\left(z_{\varepsilon}-\left(I-T_{l}\right) \varphi_{1}\right)\right\| \\
& \leq \| T_{l}^{k}\left(z_{\varepsilon}-z_{f, g}\|+\| T_{l}^{k}\left(z_{f, g}-\left(I-T_{l}\right) \varphi_{1}\right) \|\right. \\
& \leq \varepsilon+\left\|z_{f, g}-\left(I-T_{l}\right) \varphi_{k}\right\| .
\end{aligned}
$$

Substituting (21) in the last inequality, we obtain

$$
\left\|z_{\varepsilon}-\left(I-T_{l}\right) \varphi_{k+1}^{\varepsilon}\right\| \leq \varepsilon+k^{-1 / 2}\left\|\bar{\varphi}-\varphi_{1}\right\| .
$$

Since the right hand side of (22) is lower than $\mu \varepsilon$ for $k>(\mu-1)^{-2} \times$ $\left\|\bar{\varphi}-\varphi_{1}\right\|^{2} \varepsilon^{-2}$, we have $k\left(\varepsilon, z_{\varepsilon}\right) \leq c \varepsilon^{-2}$, where the constant $c>0$ depends only on $\mu$ and $\varphi_{1}$.

From Theorem 12 we obtain the desired convergence rates for the residuals in the Mann-Maz'ya iteration. (Notice that, as in Theorem 9, we do not make any additional regularity assumption on the solution $\bar{\varphi}$.) 
Corollary 13. Let $(f, g)$ be consistent Cauchy data, $\tau>1$ and $\varepsilon>0$. Given the noisy data $\left(f_{\varepsilon}, g_{\varepsilon}\right)$, with $\left\|z_{\varepsilon}-z_{f, g}\right\| \leq \varepsilon$, the stopping rule $k\left(\varepsilon, z_{\varepsilon}\right)$ determined by the discrepancy principle satisfies

i) $\left\|z_{\varepsilon}-\left(I-T_{l}\right) \varphi_{k\left(\varepsilon, z_{\varepsilon}\right)}^{\varepsilon}\right\| \leq \mu \varepsilon$;

ii) $k\left(\varepsilon, z_{\varepsilon}\right)=O\left(\varepsilon^{-2}\right)$.

\subsection{Convergence Rates Under Source Conditions}

In this section we again use the discrepancy principle as stopping rule for the iteration. However, differently from Section 4.4, we make additional regularity assumptions on the solution of the fixed point equation (3). This assumptions are stated in the form of the so-called source conditions. This is a common way to insert in the estimates some a priori knowledge about the solution and the spectrum of the operator $T_{l}$. In this way, we can also obtain convergence rates for the approximate solutions, not only for the resulting residuals.

Source conditions for linear problems usually have the form

$$
\bar{\varphi}-\varphi_{1}=f(T) \psi, \quad\|\psi\| \leq \widetilde{\psi} .
$$

If $f(\lambda)=\lambda^{\mu}$ for some $\mu>0$, we have the Hölder-type source conditions. Since our problem is exponentially ill-posed (the eigenvalues of $T_{l}$ converge exponentially to 1; see e.g. [Le]) this type of condition is too restrictive. Much more natural in this case is to use logarithmic-type source conditions:

$$
f(\lambda):= \begin{cases}\left(\ln \left(\exp (1) \lambda^{-1}\right)\right)^{-p} & \lambda>0 \\ 0 & \lambda=0\end{cases}
$$

with some parameter $p>0$. (See Remark 16 for an interpretation of this source condition.)

Theorem 14. Let $(f, g)$ be consistent Cauchy data and assume that the solution $\bar{\varphi}$ of the fixed point equation (3) satisfies the source condition

$$
\bar{\varphi}-\varphi_{1}=f\left(I-T_{l}\right) \psi, \quad \text { for some } \psi \in \mathcal{H},
$$

where $\varphi_{1} \in \mathcal{H}$ is some initial guess and $f$ is the function defined in (23) with $p \geq 1$. Let $\mu>2,\left(f_{\varepsilon}, g_{\varepsilon}\right)$ some given noisy data with $\left\|z_{\varepsilon}-z_{f, g}\right\| \leq \varepsilon, \varepsilon>0$ and $k\left(\varepsilon, z_{\varepsilon}\right)$ the stopping rule determined by the discrepancy principle. Then there exists a constant $C$, depending on $p$ and $\|\psi\|$ only, such that

$$
\begin{array}{ll}
\text { i) } & \left\|\bar{\varphi}-\varphi_{k}^{\varepsilon}\right\| \leq C(\ln k)^{-p} \\
\text { ii) } & \left\|z_{\varepsilon}-\left(I-T_{l}\right) \varphi_{k}^{\varepsilon}\right\| \leq C k^{-1}(\ln k)^{-p}
\end{array}
$$

for all iteration index $k$ satisfying $1 \leq k \leq k\left(\varepsilon, z_{\varepsilon}\right)$. 
Proof. We set $e_{k}:=\varphi_{k}^{\varepsilon}-\bar{\varphi}, P:=I-T_{l}$ (note that $P$ is self-adjoint positive non-expansive and compact). It follows from the definition of the iterative algorithm that

$$
\begin{aligned}
& e_{k}=(I-P)^{k-1} e_{1}+\sum_{j=0}^{k-2}(I-P)^{j}\left(z_{f, g}-z_{\varepsilon}\right), \\
& P e_{k}=(I-P)^{k-1} P e_{1}+(P-(I-P))^{k-1}\left(z_{f, g}-z_{\varepsilon}\right),
\end{aligned}
$$

for $k \geq 2$. Now, from Lemma A.2, follow the estimates

$$
\left\|e_{k}\right\| \leq c\|\psi\|(\ln (k+1))^{-p}+\varepsilon(k-1)
$$

and

$$
\left\|P e_{k}\right\| \leq c\|\psi\|(k-1)^{-1}(\ln (k+1))^{-p}+\varepsilon .
$$

Further, we obtain from the discrepancy principle

$$
\mu \varepsilon \leq\left\|z_{\varepsilon}-\left(I-T_{l}\right) \varphi_{k}^{\varepsilon}\right\|=\left\|P \varphi_{k}^{\varepsilon}-P \bar{\varphi}+z_{f, g}-z_{\varepsilon}\right\| \leq\left\|P e_{k}\right\|+\varepsilon .
$$

for $1 \leq k<k\left(\varepsilon, z_{\varepsilon}\right)$. It follows from (28) and (27)

$$
\varepsilon(\mu-2) \leq c\|\psi\|(k-1)^{-1}(\ln (k+1))^{-p} .
$$

Now, from (29) and (26) we obtain

$$
\left\|e_{k}\right\| \leq c\|\psi\|\left(1+\frac{1}{\mu-2}\right)(\ln (k+1))^{-p}
$$

Since $\tilde{c}:=\sup _{k \in \mathbb{N}}\left\{(\ln (k+1) / \ln (k))^{-p}\right\}<\infty$, assertion $\left.i\right)$ follows from (30) with $C=c\|\psi\|(1+1 /(\mu-2)) \tilde{c}$. Now we prove ii). It follows from (28) and (27)

$$
\left\|z_{\varepsilon}-\left(I-T_{l}\right) \varphi_{k}^{\varepsilon}\right\| \leq\left\|P e_{k}\right\|+\varepsilon \leq c\|\psi\|(k-1)^{-1}(\ln (k+1))^{-p}+2 \varepsilon
$$

and together with (29) we have

$$
\left\|z_{\varepsilon}-\left(I-T_{l}\right) \varphi_{k}^{\varepsilon}\right\| \leq c\|\psi\|\left(1+\frac{2}{\mu-2}\right)(k-1)^{-1}(\ln (k+1))^{-p} .
$$

Since $\left.c^{\star}:=\sup _{k \in \mathbb{N}}\{(k+1) / k)\right\}<\infty$, assertion ii) follows from (31) with $C=c\|\psi\|(1+1 /(\mu-2)) c^{\star} \tilde{c}$.

It is simple to check that, in the case of exact Cauchy data $(\varepsilon=0)$, assertions $i$ ) and ii) of Theorem 14 hold for every $k \geq 1$. Therefore, in the case of exact data and under the source condition (24), the iteration approximates the solution of the fixed point equation (3) with a rate of $O\left(\ln (k)^{-p}\right)$.

In the case of noisy data, the next theorem gives an estimate for the asymptotic behavior of the stopping index $k\left(\varepsilon, z_{\varepsilon}\right)$ in dependence of the noisy level $\varepsilon$. 
Theorem 15. Set $k_{\varepsilon}:=k\left(\varepsilon, z_{\varepsilon}\right)$. Under the assumptions of Theorem 14 we have

i) $k_{\varepsilon}\left(\ln \left(k_{\varepsilon}\right)\right)^{p}=O\left(\varepsilon^{-1}\right)$;

ii) $\left\|\bar{\varphi}-\varphi_{k_{\varepsilon}}^{\varepsilon}\right\| \leq O\left((-\ln \sqrt{\varepsilon})^{-p}\right)$.

Proof. In the sequence we use the notation of Theorem 14. From inequality (29) for $k=k_{\varepsilon}-1$ follows

$$
(\mu-2) \varepsilon \leq c_{1}\left(k_{\varepsilon}-2\right)^{-1}\left(\ln k_{\varepsilon}\right)^{-p}
$$

and we obtain

$$
\varepsilon^{-1} \geq c_{2}\left(k_{\varepsilon}-2\right)\left(\ln k_{\varepsilon}\right)^{p} \geq c_{3} k_{\varepsilon}\left(\ln k_{\varepsilon}\right)^{p},
$$

proving the first assertion. Now we prove ii). From (25) follows

$$
\begin{aligned}
e_{k_{\varepsilon}} & =(I-P)^{k_{\varepsilon}-1} e_{1}+\sum_{j=0}^{k_{\varepsilon}-2}(I-P)^{j}\left(z_{f, g}-z_{\varepsilon}\right), \\
& =f(P) \psi_{k_{\varepsilon}}+\sum_{j=0}^{k_{\varepsilon}-2}(I-P)^{j}\left(z_{f, g}-z_{\varepsilon}\right),
\end{aligned}
$$

where $\psi_{k_{\varepsilon}}:=(I-P)^{k_{\varepsilon}-1} \psi$. Then we can estimate

$$
\left\|e_{k_{\varepsilon}}\right\| \leq\left\|f(P) \psi_{k_{\varepsilon}}\right\|+\varepsilon k_{\varepsilon} .
$$

Next we estimate the term $P f(P) \psi_{k_{\varepsilon}}$. From (32) follows

$$
\begin{aligned}
\left\|P f(P) \psi_{k_{\varepsilon}}\right\| & =\left\|P e_{k_{\varepsilon}}-\left[\left(I-(I-P)^{k_{\varepsilon}-1}\right)\left(z_{f, g}-z_{\varepsilon}\right)\right]\right\| \\
& \leq\left\|P e_{k_{\varepsilon}}-\left(z_{f, g}-z_{\varepsilon}\right)\right\|+\varepsilon \\
& =\left\|\left(I-T_{l}\right) \varphi_{k_{\varepsilon}}^{\varepsilon}-z_{\varepsilon}\right\|+\varepsilon \\
& \leq(\mu+1) \varepsilon .
\end{aligned}
$$

Further, we have from Lemma A.3

$$
\begin{aligned}
& \left\|P f(P) \psi_{k_{\varepsilon}}\right\|^{2} \\
& \quad=\exp (1)^{-2} \int_{0}^{1} \exp \left(-\left[(1-\ln (\lambda))^{-2 p}\right]^{-1 /(2 p)}\right)^{2}(1-\ln (\lambda))^{-2 p} d\left\|E_{\lambda} \psi_{k_{\varepsilon}}\right\|^{2} \\
& \quad \geq \exp (1)^{-2} \hat{h}\left(\int_{0}^{1}(1-\ln (\lambda))^{-2 p} d\left\|E_{\lambda} \psi_{k_{\varepsilon}}\right\|^{2}\right) \\
& \quad=\exp (1)^{-2} \hat{h}\left(\left\|f(P) \psi_{k_{\varepsilon}}\right\|^{2}\right) .
\end{aligned}
$$

Then we obtain from (34), (35) and Lemma A.4

$$
\left\|f(P) \psi_{k_{\varepsilon}}\right\| \leq O\left(\left(-\ln \left(\varepsilon^{2 / 3}\right)\right)^{-p}\right) .
$$


Now we estimate the term $\varepsilon k_{\varepsilon}$. From assertion $i$ ) and Lemma A.5 follows

$$
k_{\varepsilon}=O\left(\varepsilon^{-1}(-\ln \sqrt{\varepsilon})^{-p}\right) .
$$

Now, from (33), (36), (37) follows

$$
\left\|e_{k_{\varepsilon}}\right\| \leq O\left(\left(-\ln \left(\varepsilon^{2 / 3}\right)\right)^{-p}\right)+\varepsilon O\left(\varepsilon^{-1}(-\ln \sqrt{\varepsilon})^{-p}\right) \leq O\left((-\ln \sqrt{\varepsilon})^{-p}\right),
$$

proving the second assertion.

Remark 16. Next we consider the question of how to interpret the source condition defined in (24). Let $\Omega$ be the square $[-\pi, \pi] \times[-\pi, \pi], \quad \Gamma_{1}=$ $\{(x, y) \in \partial \Omega ; x=-\pi\}, \quad \Gamma_{2}=\{(x, y) \in \partial \Omega ; x=\pi\}$ and consider the Cauchy problem

$$
\begin{cases}\Delta u=0 & \text { in } \Omega \\ u=f & \text { at } \Gamma_{1} \\ u_{v}=g & \text { at } \Gamma_{1} \\ u(x, \pm \pi)=0 & x \in(-\pi, \pi)\end{cases}
$$

where $f(y)=\sum_{j=1}^{N} a_{j} \sin (j y), g(y)=\sum_{j=1}^{N} b_{j} \sin (j y)$. Given the Neumann data $\varphi(y)=\sum_{j=1}^{N} \varphi_{j} \sin (j y)$, we can explicitly represent the operator $T$ defined in (2) by

$$
(T \varphi)(y)=\sum_{j=1}^{N}\left[\left(\frac{\alpha_{j}}{\beta_{j}}\right)^{2} \varphi_{j}+\left(\frac{j \alpha_{j}}{\beta_{j}^{2}}\right) a_{j}+\frac{1}{\beta_{j}} b_{j}\right] \sin (j y),
$$

where $\alpha_{j}=\sinh (2 j \pi)$ and $\beta_{j}=\cosh (2 j \pi)$. Now we define the Sobolev spaces of periodic functions

$$
H_{\mathrm{per}}^{s}(-\pi, \pi):=\left\{\varphi(y)=\sum_{j \in \mathbb{Z}} \varphi_{j} e^{i j y} \mid \sum_{j \in \mathbb{Z}}\left(1+j^{2}\right)^{s} \varphi_{j}^{2}<\infty\right\}, \quad s \in \mathbb{R} .
$$

If the Cauchy data $(f, g)$ is sufficiently regular, the Maz'ya iteration is well defined at $H_{\mathrm{per}}^{-1 / 2}\left(\Gamma_{1}\right)$ and we obtain from (38) a spectral representation of the linear part of the operator $T$ :

$$
\left(T_{l} \varphi\right)(y)=\sum_{j=1}^{\infty} \lambda_{j} \varphi_{0, j} \sin (j y)
$$

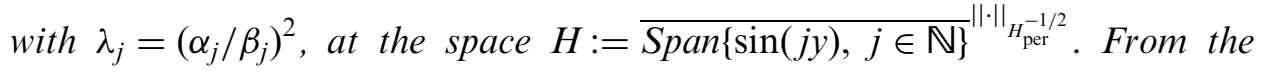
estimate

$$
\begin{aligned}
\ln \left(\frac{\exp (1)}{1-\lambda_{j}}\right) & \geq 1-\ln \left(\exp (1)\left[1-\frac{\alpha_{j}}{\beta_{j}}\right]\right) \\
& =-\ln \left(\frac{2 \exp (-2 j \pi)}{\exp (2 j \pi)+\exp (-2 j \pi)}\right) \geq 4 \pi j-1
\end{aligned}
$$


and from the source condition (24) follows

$$
\begin{aligned}
\left\|\bar{\varphi}-\varphi_{1}\right\|_{p}^{2} & =\sum_{j=1}^{\infty}\left(1+j^{2}\right)^{p} \ln \left(\frac{\exp (1)}{1-\lambda_{j}}\right)^{-2 p} \psi_{j}^{2} \\
& \leq \sum_{j=1}^{\infty}\left(1+j^{2}\right)^{p}(4 \pi j-1)^{-2 p} \psi_{j}^{2} \leq c \sum_{j=1}^{\infty} \psi_{j}^{2}<\infty .
\end{aligned}
$$

This means that the source condition (24) can be interpreted as a regularity condition in the sense of $H^{p}$ spaces, i.e. $\bar{\varphi}-\varphi_{1} \in H_{\mathrm{per}}^{p}\left(\Gamma_{1}\right)$. This shows that a logarithmic source condition is indeed appropriate for our problem.

\section{NUMERICAL EXPERIMENTS}

Next we present some numerical results related to the numerical implementation of the Mann-Maz'ya iteration. The first two problems concern consistent Cauchy problems in a square and in an annular domain. In the third example we consider a problem with noisy data.

The computation was performed on the Silicon Graphics SGI-machines (based on R12000 processors; 32-bit code) at the Spezialforschungsbereich F013. The elliptic mixed boundary value problems were solved using the (NETLIB) software package PLTMG. ${ }^{9}$

\subsection{A Consistent Problem in a Rectangular Domain}

Let $\Omega \subset \mathbb{R}^{2}$ be the open rectangle $(0,1) \times(0,3 / 4)$ and define the following subsets of $\partial \Omega$ :

$$
\begin{array}{ll}
\Gamma_{1}:=\{(x, 0) ; x \in(0,1)\}, & \Gamma_{2}:=\{(x, 3 / 4) ; x \in(0,1)\}, \\
\Gamma_{3}:=\{(0, y) ; y \in(0,3 / 4)\}, & \Gamma_{4}:=\{(1, y) ; y \in(0,3 / 4)\} .
\end{array}
$$

We consider the Cauchy problem

$$
\begin{cases}\Delta u=0 & \text { in } \Omega \\ u=f & \text { at } \Gamma_{1} \\ u_{v}=g & \text { at } \Gamma_{1} \\ u=0 & \text { at } \Gamma_{3} \cup \Gamma_{4}\end{cases}
$$

where the Cauchy data $f(x)=\sin (\pi x), g \equiv 0$ is given at $\Gamma_{1}$. We aim to reconstruct the (Dirichlet) trace of $u$ at $\Gamma_{2}$. As one can easily check, the exact solution of this Cauchy Problem is given by $\bar{u}(x, y)=\cosh (\pi y) \sin (\pi x)$.

\footnotetext{
${ }^{9}$ See URL http://www.netlib.org for details.
} 
We used in the iteration the matrix $A=\left(a_{j k}\right)$, with $a_{j k}=k^{-1}$ for $j \leq k$. As initial guess, we chose $\varphi_{1} \equiv 0$. Each mixed boundary value problem was solved using (multi-grid) finite element methods, with linear elements and a uniform mesh with 65921 nodes (256 nodes on $\Gamma_{2}$ ). We used the stopping rule $\left\|\varphi_{k}-\varphi_{k-1}\right\|_{L^{2}\left(\Gamma_{2}\right)} \leq 10^{-3}$. In Figure 1 we present the results corresponding to the Mann-Maz'ya iteration: the dotted line represents the exact solution and the solid line represents the sequence generated by $(0, A, T)$.

As one can observe in Figure 1, the convergence rate decays very fast. This can be in part explained by linear convex combination used to compute $\psi_{k}$ in the Mann-Maz'ya iteration (note that $\psi_{k+1}-\psi_{k}=(1 /(k+1)) \times$ $\left.\varphi_{k+1}-(1 / k(k+1)) \sum_{j=1}^{k} \varphi_{k}\right)$. As an alternative to improve the convergence rate, we relaxed the stopping rule and restarted the iteration, using the last evaluated $\varphi_{k}$ as new initial guess. In Figure 2 we present the results corresponding to this restart strategy. For comparison purposes, we restarted the iteration after every 50 steps. Thus, to compute the results in Figure 2, we had to evaluate $50,100,250$ and 500 iteration steps respectively.

The restart strategy seams to save a considerable amount of computation effort, however we should quote that we have no analytical justification neither for the choice of the restart criterion nor for the improvement in the convergence rate. (Argumenting as in Section 4.3, one can verify that the sequence of residuals is again non-increasing.)
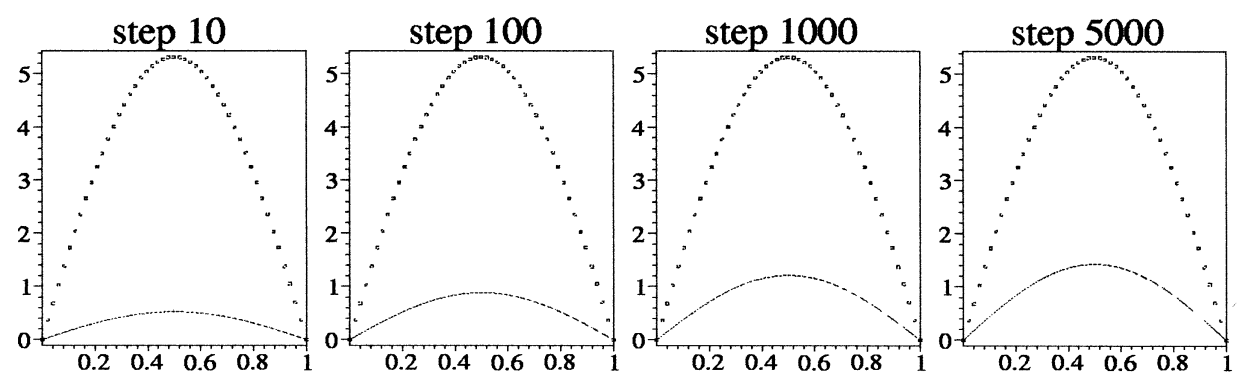

Figure 1. Rectangular domain; iteration for consistent Cauchy data.
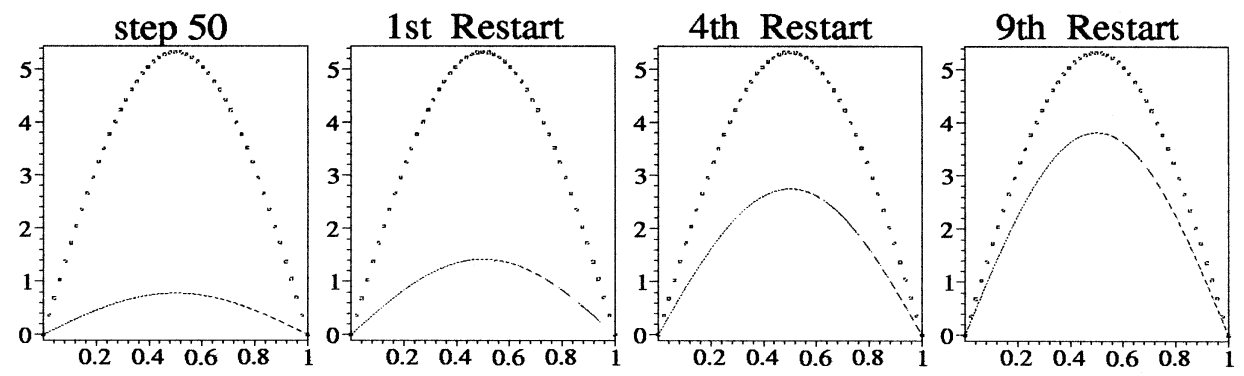

Figure 2. Rectangular domain; iteration with restart strategy. 


\subsection{A Consistent Problem in an Annular Domain}

Let $\Omega$ be the annulus centered at the origin with inner and outer radius respectively 1 and 3 . We denote the inner and outer boundaries by $\Gamma_{1}$ and $\Gamma_{2}$ respectively. We consider the following Cauchy problem:

$$
\begin{cases}\Delta u=0 & \text { in } \Omega \\ u=f & \text { at } \Gamma_{1} \\ u_{v}=g & \text { at } \Gamma_{1}\end{cases}
$$

Given the Cauchy data $f(\theta)=\sin (\theta)-\sin (2 \theta) / 2$ and $g \equiv 0$ at $\Gamma_{1}$, we want to reconstruct at $\Gamma_{2}$ the trace of $u$. It is easy to check, that the solution of this Cauchy problem is given by $\bar{u}(r, \theta)=(1 / 2)\left(r+r^{-1}\right)$ $\sin (\theta)-1 / 4\left(r^{2}+r^{-2}\right) \sin (2 \theta)$.

The matrix $A$ is chosen as in Section 5.1. For the multi-grid method we used linear elements and a (uniform) mesh with 61824 nodes (512 nodes on $\Gamma_{2}$ ). We use the same initial guess $\varphi_{1} \equiv 0$ and the same stopping rule as in the previous example. In Figure 3 we present the results corresponding to the Mann-Maz'ya iteration: the dotted line represents the exact solution and the solid line represents the sequence generated by the iteration $(0, A, T)$. (Note that in Figures $3-5$ the horizontal axis is parameterized from 0 to $2 \pi$.)
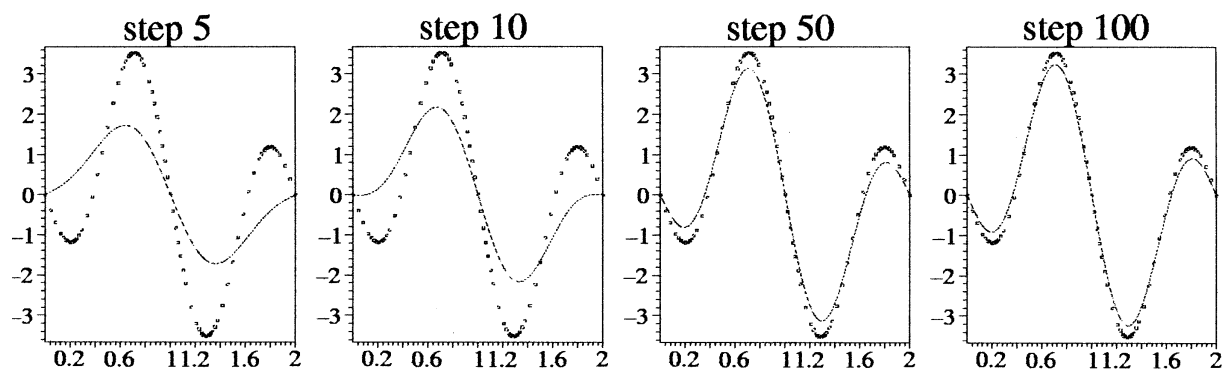

Figure 3. Annular domain; iteration for consistent Cauchy data.

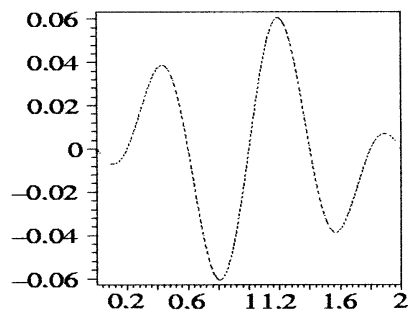

(a)

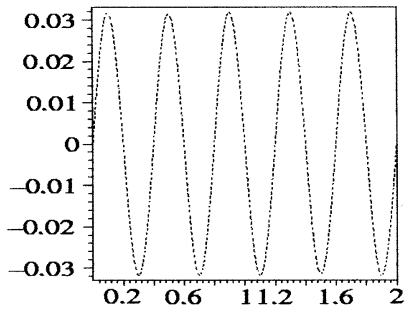

(b)

Figure 4. Generation of noisy data; (a) Perturbation added to the Dirichlet data; (b) Perturbation added to the Neumann data. 

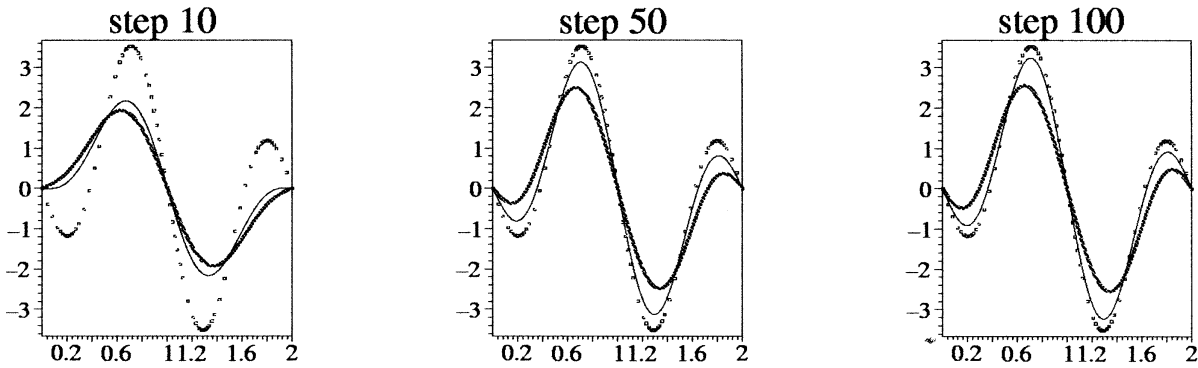

Figure 5. Iteration for noisy data in the annular domain; dotted-line: exact solution; thinline: iteration for exact data; thick-line: iteration for noisy data.

\subsection{An Inconsistent Problem (Noisy Data)}

For this experiment we consider once more the Cauchy problem formulated in Section 5.2. The noisy data is obtained by inserting in the exact Cauchy data $(f, g)=(\sin (\theta)-(1 / 2) \sin (2 \theta), 0)$ a perturbation of $5 \%$. In Figure 4 we present the perturbations added to the Dirichlet and to the Neumann data.

The matrix $A$ is chosen as in Section 5.1. The initial guess, stopping rule and mesh level used for the computation are the same as those considered in that section. The results corresponding to the Mann-Maz'ya iteration are presented in Figure 5 (thick line).

As one can see in Figure 5, the performance of the numerical implementation of the Mann-Maz'ya iteration is stable. The high frequency components of the error start to interfere in the iteration only after an exponential number of steps. Indeed, in the Maz'ya algorithm the iteration error $e_{k}:=\bar{\varphi}-\varphi_{k}^{\varepsilon}$ satisfies $e_{k+1}=T_{l} e_{k}=T_{l}^{k} e_{1}$, and the eigenvalues of the fixed point operator $T_{l}$ converge exponentially to 1 (see Remark 16 for an example). Thus, the iteration reconstructs first the projection of the solution over the first eigenspace of the operator $T_{l}$; after an exponential number of steps, it reconstructs also the projection over the second eigenspace; and so on.

The high frequency components of the error are exponentially amplified and destroy completely the approximation if we iterate long enough. However, due to the characteristic explained above, one has to evaluate an exponential number of steps in order to observe the influence of the bad frequencies.

\section{FINAL REMARKS}

Let us suppose $\partial \Omega=\Gamma_{1} \cup \Gamma_{2} \cup \Gamma_{3}$ and we want to analyze a Cauchy problem with data given at $\Gamma_{1}$ plus some further boundary condition (Neumann, Dirichlet, ...) at $\Gamma_{3}$ (this is precisely the situation in Section 5.1). 
It is still possible to use the Maz'ya method for such problems. All we have to do is to adapt the Maz'ya iteration by adding this extra boundary condition at $\Gamma_{3}$ to both mixed boundary value problems at each iteration step. This over-determination of boundary data does not affect the analysis of the Maz'ya algorithm (see [Le]). Consequently, it also does not affect the analysis of the Mann-Maz'ya iteration presented here.

The Maz'ya iterative method (see Section 3.1) generates a sequence of Neumann traces, which approximate the unknown Neumann boundary condition $u_{\left.v_{A}\right|_{\Gamma_{2}}}$. Analogously, we can define an iterative method, which produces a sequence of Dirichlet traces (this was already suggested by Maz'ya et al. in [KMF]). The convergence proof for this iteration is quite similar to the one presented in [Le] for the Maz'ya iteration. It is also possible to combine this iteration with the Mann strategy. All the results formulated here for the Mann-Maz'ya method remain valid for this iteration.

The approach followed in [Le] to characterize the solution of elliptic Cauchy problems as a solution of a fixed point equation can be extended, using spectral theory, to differential operators of hyperbolic and parabolic types (see $[\mathrm{BaLe}]$ ). The formulation of the Mann iteration for this problems follow the lines discussed here.

\section{APPENDIX}

Lemma A.1. Let $p>0$ and $k \in \mathbb{N}_{0}$. Define the real-valued function $\hat{f}(\lambda):=(1-\lambda)^{k}(\ln (\exp (1)) / \lambda)^{-p}, \lambda \in[0,1]$. Then we have

$$
\hat{f}(\lambda) \leq C(\ln k)^{-p}, \quad \lambda \in[0,1],
$$

with $C$ independent of $k$. Moreover, for each $p \in \mathbb{R}$, the real-valued function $\hat{g}(\lambda):=(1-\lambda)^{k} \lambda(\ln (\exp (1)) / \lambda)^{-p}$ defined on $[0,1]$, satisfies

$$
\hat{g}(\lambda) \leq C k^{-1}(\ln k)^{-p}, \quad \lambda \in[0,1],
$$

with $C$ independent of $k$.

Proof. The first assertion is proved in [DES, Lemma A.1]. The second assertion is quite similar to the second part of the lemma cited above and can be proved with an analogous argumentation.

Lemma A.2. Let $p>0, \mathcal{H}$ a Hilbert space, $P: \mathcal{H} \rightarrow \mathcal{H}$ a positive linear self adjoint non-expansive operator, $f$ the real-valued function defined by

$$
f(\lambda):= \begin{cases}\left(\ln \left(\exp (1) \lambda^{-1}\right)\right)^{-p} & \lambda \in(0,1] \\ 0 & \lambda=0 .\end{cases}
$$


Let $e_{1}:=f(P) \psi$, for some $\psi \in \mathcal{H}$. Then for any $k>1$

$$
\begin{aligned}
\left\|(I-P)^{k} e_{1}\right\| & \leq C\|\psi\|(\ln (k+2))^{-p} \\
\left\|(I-P)^{k} P e_{1}\right\| & \leq C\|\psi\| k^{-1}(\ln (k+2))^{-p}
\end{aligned}
$$

with $C$ independent of $k$.

Proof. This lemma is analogue to the result stated in [DES, Lemma 2.6]. It follows from Lemma A.1, the same way as [DES, Lemma 2.6] follows form [DES, Lemma A.1].

Lemma A.3. Given $p>1$, then

$$
\int_{0}^{1} \hat{h}\left((1-\ln (\lambda))^{-2 p}\right) d \lambda \geq \hat{h}\left(\int_{0}^{1}(1-\ln (\lambda))^{-2 p} d \lambda\right)
$$

with $\hat{h}(t):=\exp \left(-t^{(-1 / 2 p)}\right)^{2} t$.

Proof. The assertion follows from the convexity of $\hat{h}$ in $[0, \infty)$ and Jensen's inequality. (see [DES, Lemma A.2])

Lemma A.4. Let $p \geq 1, C>0$ and $\varepsilon>0$ sufficiently small such that $1 \geq$ $\left(-\ln \left(C \varepsilon^{2 / 3}\right)\right)^{-2 p} \geq \varepsilon$. Let

$$
\int_{0}^{1} \hat{h}\left((1-\ln (\lambda))^{-2 p}\right) d\left\|E_{\lambda} \psi\right\|^{2}=C \varepsilon^{2}
$$

Then there exists $D>0$ (independent of $\varepsilon$ ) such that

$$
\int_{0}^{1}(1-\ln (\lambda))^{-2 p} d\left\|E_{\lambda} \psi\right\|^{2} \leq D\left(-\ln \left(\varepsilon^{2 / 3}\right)\right)^{-2 p} .
$$

Proof. Let $s_{2}=\left(-\ln \left(C \varepsilon^{2 / 3}\right)\right)^{-2 p}$. Then

$$
\hat{h}\left(s_{2}\right)=\left(C \varepsilon^{2 / 3}\right)^{2}\left(-\ln \left(C \varepsilon^{2 / 3}\right)\right)^{-2 p} \geq C \varepsilon^{2} .
$$

Thus, it follows from the monotonicity of $\hat{h}$ that the equation $\hat{h}(s)=C \varepsilon^{2}$ has a solution $s_{1} \in\left(0, s_{2}\right]$. From the assumptions and Lemma A.3 follows

$$
\hat{h}\left(\int_{0}^{1}(1-\ln (\lambda))^{-2 p} d\left\|E_{\lambda} \psi\right\|^{2}\right) \leq C \varepsilon^{2}
$$

and we conclude from the monotonicity of $\hat{h}$ that

$$
\int_{0}^{1} \hat{h}\left((1-\ln (\lambda))^{-2 p}\right) d\left\|E_{\lambda} \psi\right\|^{2} \leq s_{1} \leq s_{2} \leq D\left(-\ln \left(\varepsilon^{2 / 3}\right)\right)^{-2 p}
$$

for a generic constant $D$.

Lemma A.5. Let $\hat{k}$ be a solution of

$$
k(\ln k)^{p}=C \varepsilon^{-1} \text {. }
$$


Then $\hat{k}$ satisfies

$$
\hat{k}=O\left(\varepsilon^{-1}(-\ln \sqrt{\varepsilon})^{-p}\right) .
$$

Proof. Follows immediately from [DES, Lemma A.6].

\section{REFERENCES}

[Ad] Adams, R. Sobolev Spaces, Academic Press, New York, 1975.

[Ba] Baumeister, J. Stable Solution of Inverse Problems, Friedr. Vieweg \& Sohn, Braunschweig, 1987.

[BaLe] Baumeister, J.; Leitão, A. On iterative methods for solving ill-posed problems modeled by partial differential equations, J. Inverse Ill-Posed Probl., 9 (2001), 13-29.

[BrPe] Browder, F.; Petryshyn, W. Construction of fixed points of nonlinear mappings in Hilbert space, J. Math. Anal. Appl. 20 (1967), 197-228.

[DaLi] Dautray, R.; Lions, J.L. Mathematical Analysis and Numerical Methods for Science and Technology, Vol. 2: Functional and Variational Methods, Springer-Verlag, New York, 1988.

[DES] Deuflhard, P.; Engl, H.W.; Scherzer, O. A convergence analysis of iterative methods for the solution of nonlinear ill-posed problems under affinely invariant conditions, Inverse Problems, 14 (1998), 1081-1106.

[Do] Dotson, W. On the Mann iterative process, Trans. Am. Math. Soc. 149 (1970), 65-73.

[EnSc] Engl, H.W.; Scherzer, O. Convergence rates for iterative methods for solving nonlinear ill-posed problems, In: Surveys on Solution Methods for Inverse Problems; D. Colton et al. (Eds.), 7-34, Springer, Vienna, 2000.

[EHN] Engl, H.W.; Hanke, M.; Neubauer, A. Regularization of Inverse Problems, Kluwer Academic Publishers, Dordrecht, 1996 (Paperback: 2000).

[GiTr] Gilbarg, D.; Trudinger, N. Elliptic Partial Differential Equations of Second Order, Springer-Verlag, New York, 1977.

[Gr] Groetsch, C. A note on segmenting Mann iterates, J. Math. Anal. Appl. 40 (1972), 369-372.

[Had] Hadamard, J. Le problème de Cauchy et les équations aux dérivées partielles linéaires hyperboliques, Hermann, Paris, 1932.

[Is] Isakov, V. On the uniqueness of the solution of the Cauchy problem, Soviet Math. Dok1. 22 (1980), 639-642.

[Je] Jeggle, H. Nichtlineare Funktionalanalysis, Teubner, Stuttgart, 1979.

[KMF] Kozlov, V.; Maz'ya, V.; Fomin, A. An iterative method for solving the Cauchy problem for elliptic equations, Comput. Math. Math. Phys. 31 (1991), 45-52.

[Le] Leitão, A. An iterative method for solving elliptic Cauchy problems, Numer. Funct. Anal. Optim. 21 (2000), 715-742.

[Ma] Mann, W. Mean value methods in iteration, Proc. Amer. Math. Soc. 4 (1953), 506-510. 


\section{Request Permission or Order Reprints Instantly!}

Interested in copying and sharing this article? In most cases, U.S. Copyright Law requires that you get permission from the article's rightsholder before using copyrighted content.

All information and materials found in this article, including but not limited to text, trademarks, patents, logos, graphics and images (the "Materials"), are the copyrighted works and other forms of intellectual property of Marcel Dekker, Inc., or its licensors. All rights not expressly granted are reserved.

Get permission to lawfully reproduce and distribute the Materials or order reprints quickly and painlessly. Simply click on the "Request

Permission/Reprints Here" link below and follow the instructions. Visit the U.S. Copyright Office for information on Fair Use limitations of U.S. copyright law. Please refer to The Association of American Publishers' (AAP) website for guidelines on Fair Use in the Classroom.

The Materials are for your personal use only and cannot be reformatted, reposted, resold or distributed by electronic means or otherwise without permission from Marcel Dekker, Inc. Marcel Dekker, Inc. grants you the limited right to display the Materials only on your personal computer or personal wireless device, and to copy and download single copies of such Materials provided that any copyright, trademark or other notice appearing on such Materials is also retained by, displayed, copied or downloaded as part of the Materials and is not removed or obscured, and provided you do not edit, modify, alter or enhance the Materials. Please refer to our Website User Agreement for more details.

\section{Order now!}

Reprints of this article can also be ordered at http://www.dekker.com/servlet/product/DOI/101081NFA100108313 